\title{
Las elecciones de 2012: hechos y números
}

\section{Sergio García Ramírez \\ Mayarí Forno Oliva}

\section{Sumario:}

I. Introducción

II. El personal y la estructura del IFE

III. Plan Integral

IV. Recursos financieros

V. El padrón electoral y la lista nominal

VI. Cartografía electoral y disposición de casillas

VII. Documentación y material electoral

VIII. Funcionarios de casillas y colaboradores

IX. Representantes de partidos políticos ante las casillas

$X$. Observadores electorales y visitantes

XI. La jornada electoral

XII. Resultados electorales

XIII. Declaración de validez y asignación de curules

XIV. Declaración de validez y cómputo final de la elección de presidente

$X V$. Reflexiones finales

$X V I$. Fuentes 


\section{Introducción}

El proceso electoral de 2011-2012 constituye un nuevo capítulo en el largo camino de la democracia formal en México. En el curso del siglo $\mathrm{XX}$ se plantearon otros capítulos, a intervalos largos, que contribuyeron a esta marcha: elección directa de representantes populares, voto activo y pasivo de las mujeres, ciudadanía de la juventud, fortalecimiento de los partidos políticos, representación proporcional, en distintas etapas y con diversas manifestaciones.

Este ha sido el paso de la democracia electoral, en la que se concentra el presente artículo. Queda fuera de nuestro tema actual la otra vertiente de la democracia, social o integral. En esta vertiente, la democracia se constituye como un sistema de vida fundado en el constante mejoramiento del pueblo, tal como se expresa en el artículo 30. de la Constitución Política, tema que amerita reflexión separada que examine - como se ha hecho reiteradamente- las grandes y graves deficiencias que aún prevalecen en este ámbito.

La democracia formal o electoral - que algunos designan como "democracia sin adjetivos" - ha recogido elementos sobresalientes que contribuyen a establecer su fisonomía contemporánea en nuestro país. Sin pretender agotar la relación de esos elementos, reunidos en el largo camino al que nos referimos, mencionaremos solamente la notable extensión de la base popular del poder, mediante la ampliación del derecho al voto activo o pasivo. Por un lado, la presencia y operación de diversas corrientes políticas, formalizadas en partidos, que reflejan las condiciones de una sociedad plural, mejor informada, deliberante y participativa. Por otro lado, la organización, fiscalización y calificación de las elecciones a cargo de órganos autónomos, constitucionalmente previstos y garantizados. Finalmente la frecuente variación de la normativa electoral, cada vez más abundante y detallada, para atender las exigencias que provienen de nuevas circunstancias, problemas emergentes y expectativas de progreso democrático.

El 1o. de julio de 2012 se desarrolló la jornada electoral federal más concurrida de la historia de México. Acudieron a las urnas 50143616 ciudadanos, que representan el $63.10 \%$ de la lista nominal del electores, y que sufragaron en 143130 casillas. La votación fue recibida por 568948 funcionarios de casilla, en presencia de 551521 representantes de partidos políticos. Así se hizo la elección del presidente de la Repú- 
blica (periodo 2012-2018) y se integró el Congreso de la Unión. Este trabajo no pretende aportar consideraciones históricas, interpretaciones políticas o propuestas normativas, sino dar cuenta de los números principales del proceso electoral, que ilustran sobre el desarrollo de éste, y los resultados obtenidos en la jornada del 1o. de julio de 2012.

Obviamente, todos los actos del proceso electoral, desde las etapas de preparación hasta la culminación provista por la sentencia del Tribunal Electoral del Poder Judicial de la Federación, del 31 de agosto de 2012, se hallan sujetos a riguroso análisis desde diversas perspectivas. En el proceso quedaron de manifiesto coincidencias y diferencias entre sus principales actores. Los órganos electorales del Estado mexicano - Instituto Federal Electoral y Tribunal Electoral- aportaron decisiones y explicaciones. Los analistas han emitido puntos de vista diversos y numerosos, que contribuyen a establecer las características de este gran proceso. El debate está abierto. No pretendemos terciar, sino informar a partir de datos que provienen de fuentes oficiales.

La elección de presidente y de senadores de la República de 2012 fue la primera de su género en que se aplicó la legislación constitucional y secundaria expedida en 2007-2008, que anteriormente fue aplicada solamente en la elección de diputados federales en 2009. En los términos de la regulación constitucional y secundaria vigente en 20112012, el proceso electoral, sujeto a los principios rectores de certeza, legalidad, independencia, imparcialidad y objetividad, se compone de varias etapas. Cada una requiere intenso trabajo de preparación y realización, con notable despliegue de recursos de diverso género y concurrencia de múltiples actores políticos y sociales.

\section{El personal y la estructura del IFE}

La necesidad de contar con recursos humanos suficientes y competentes obliga a poner especial acento en el reclutamiento y la capacitación del personal. La logística del proceso es muy compleja y debe satisfacer demandas puntuales: accesibilidad, libertad, oportunidad, secreto de la votación, entre otras. Asimismo, considera temas vinculados a la planeación y la administración electorales y al uso de tecnología. No sobra mencionar que diversos extremos del andamiaje electoral se han organizado al amparo de una legislación dominada por la desconfian- 
za, que trae consigo procedimientos rigurosos, diversas medidas de seguridad, verificaciones, fiscalizaciones, acciones de transparencia y vigilancia con intervención de autoridades, ciudadanos y partidos políticos.

La columna vertebral de la organización de los comicios, en lo que toca al Instituto Federal Electoral, se halla en la estructura de este organismo, que se despliega en dos grandes vertientes: a) central y concentrada, con el Consejo General, la Secretaría Ejecutiva y las direcciones ejecutivas, y $b$ ) desconcentrada, con los consejos locales y distritales y las juntas ejecutivas. Con vista en los comicios de 2012, el Instituto procedió a la integración de los consejos locales (32, con participación de 192 ciudadanos: 50.52\% mujeres y 48.95\% varones) y Distritales (300, con participación de 1800 ciudadanos: $49.89 \%$ mujeres y $50.11 \%$ varones $^{1}$ ), mediante selección cuidadosa de sus integrantes. El Consejo General aprobó la integración de los consejos locales, y éstos actuaron en la integración de los consejos distritales. En el curso del proceso y para los fines de éste fue preciso ajustar y fortalecer la relación institucional entre las áreas centrales y desconcentradas, cuidando su adecuada convergencia en la preparación y realización de las elecciones.

\section{Plan Integral}

Las bases y criterios para la organización de las elecciones de 2012 se establecieron en el Plan Integral del Proceso Electoral (PIPEF) 20112012, aprobado por el Consejo General del IFE el 22 de agosto de 2011. Entre los objetivos estratégicos mencionados en el Plan figuraban: consolidar la credencial para votar como medio preferente de identidad ciudadana e incrementar la eficacia en la organización de los procesos electorales federales, la calidad del padrón electoral y la eficiencia de los procesos sustantivos. Cada objetivo se tradujo en líneas de acción específicas. $^{2}$

1 Información proporcionada por la Dirección Ejecutiva de Organización Electoral del Instituto Federal Electoral.

${ }^{2}$ Cfr. Plan Integral del Proceso Electoral 2011-2012, pp. 9 y 10, http://www.ife.org.mx/ docs/IFE-v2/ProcesosElectorales/CIPEF-PIPEF/CIPEF-PIPEF_docs/PIPEF_2011-2012. $p d f$. 
El Plan estableció indicadores que acompañaron el desarrollo de las estrategias, bajo un criterio de transparencia que permitiera a los ciudadanos y a los partidos políticos conocer las principales actividades inherentes a la organización de las elecciones. El IFE publicó mensualmente los informes de avance en la aplicación del referido Plan Integral.

\section{Recursos financieros}

Por lo que hace a recursos financieros, conviene mencionar que el presupuesto total destinado al IFE para el ejercicio de 2012 fue de 15953.9 millones de pesos. De esta cifra, 10661 millones corresponden al gasto de operación del Instituto. Estuvieron directamente asignados al proceso electoral 4938.5 millones, y 107.1 millones al programa de voto de los mexicanos residentes en el extranjero. La suma asignada a los partidos políticos bajo el concepto de prerrogativas previstas en la ley electoral fue de 5292.5 millones de pesos.

Una parte sustancial del gasto de operación se destina a satisfacer necesidades inherentes a la logística electoral: contratación de personal eventual para capacitar a los ciudadanos que fungieron como funcionarios de casillas, informar sobre el desarrollo de la jornada electoral y contribuir al conteo rápido; actualizar la infraestructura informática, instalar casillas electorales y producir los documentos y materiales que se utilizan en la jornada electoral (mamparas, boletas, urnas, actas, líquido indeleble).

\section{El padrón electoral y la lista nominal}

Un elemento de importancia vital para los procesos electorales es el padrón de ciudadanos, del que proviene la lista de electores. Se ha trabajado intensamente, a lo largo de muchos años, en el mejoramiento del padrón, a fin de que constituya un instrumento confiable para la sociedad, y desde luego para los propios partidos políticos. El Registro de Electores, cada vez mejor equipado, está sujeto a la permanente vigilancia de los partidos. Las constantes actividades inherentes a la 
dotación de credenciales a los nuevos ciudadanos, la actualización del padrón electoral, la renovación de las credenciales y otras diligencias de carácter permanente y temporal requerirían una amplia información que no podemos ofrecer en el espacio de este artículo. Para la elección federal de 2012, la lista nominal de electores definitiva con fotografía quedó integrada con 79454802 ciudadanos.

Es relevante mencionar que se instaló un comité de especialistas con el encargo de verificar la calidad de la lista nominal de electores. El comité que intervino en el proceso de 2012 determinó lo siguiente:

Considerando que ha quedado acreditada la congruencia externa de la Lista Nominal de Electores respecto de los instrumentos registrales de otras autoridades y de la dinámica democrática del país; que la Verificación Nacional Muestral es un método válido para evaluar los instrumentos registrales del Instituto Federal Electoral y que sus indicadores también reflejan la dinámica demográfica de la población mexicana; que los programas de depuración del Padrón Electoral y de la Lista Nominal de Electores responden a la intensa movilidad interna y externa de la población mexicana; que la distribución territorial de los Módulos de Atención Ciudadana es eficiente y equitativa, y que éstos cuentan con una estructura informática adecuada, se puede concluir que el nivel de precisión que ofrece el Padrón Electoral y la Lista Nominal de Electores refleja en alto grado de certidumbre las condiciones de la población registrada, y que los datos no muestran que estos instrumentos registrales pudieran contener sesgos que vulneren su confiabilidad. ${ }^{3}$

\section{Cartografía electoral y disposición de casillas}

La lista nominal y la cartografía electoral son elementos operativos esenciales para determinar el número y la localización de las casillas destinadas a la recepción de votos durante la jornada electoral. En el desarrollo de este trabajo se hizo un recorrido de secciones en todo

Acuerdo del Consejo General del Instituto Federal Electoral del 24 de mayo de 2012, por el que se declara que el padrón electoral y los listados nominales de electores que serán utilizados en la jornada electoral del 1o. de julio de 2012 son válidos y definitivos, y que la lista nominal de electores residentes en el extranjero es válida. 
el territorio nacional y se atendió a las observaciones presentadas por los partidos políticos. Desde luego, fue necesario recabar la anuencia de los ocupantes de sitios seleccionados para la instalación de las casillas.

Recordemos que en cada sección electoral se debe instalar una casilla básica para recibir la votación de 750 ciudadanos inscritos en la lista nominal. Si se rebasa este número, es preciso instalar las casillas contiguas que resulten necesarias. Puede haber, además, casillas extraordinarias, y hasta 5 casillas especiales en cada distrito para la recepción del voto de los electores que se encuentren transitoriamente fuera de la sección correspondiente a su domicilio.

Para los comicios del 1o. de julio de 2012 se aprobó la instalación de 143132 casillas, de las cuales 66526 correspondieron a la categoría de casillas básicas (46.48\%), 69602 a contiguas (48.63\%), 6102 a extraordinarias $(4.26 \%)$, y 902 a especiales $(0.63 \%)$. Es preciso tomar en cuenta - lo que no ocurre con frecuencia en el análisis de las jornadas electorales- el ingente trabajo que implica la definición del número y localización de las casillas, la dotación del material correspondiente, la instalación y el desmontaje.

\section{Documentación y material electoral}

Vayamos ahora a la documentación electoral y a otros elementos indispensables para la recepción y cómputo de sufragios. Obviamente, este rubro demanda diversas tareas de producción, inspección, verificación, traslado y custodia, en las que participan millares de personas en diversos puntos del país.

Cada casilla debe contar - y efectivamente contó- con listados nominales, boletas y actas electorales, documentación de apoyo, urnas, mamparas, líquido indeleble, marcadoras de credencial de elector, lápices, documentos y materiales especiales para capacidades diferentes, y carteles y trípticos de información para la ciudadanía. El Consejo General del IFE aprobó la documentación electoral el 25 de agosto de 2011, mediante acuerdo CG248/2011.

Se puso especial cuidado en la adopción de medidas de seguridad para evitar la falsificación de boletas: "papel seguridad", marca de agua, fibras ópticas visibles, fibras ópticas ocultas, pantalla de sello 
de agua impresa, impresión invertida e imagen latente perceptible a través de una mica especial. Además, las boletas quedaron adheridas a un talón foliado con información sobre la entidad federativa, distrito electoral, tipo de elección y número progresivo de folio. Se imprimieron 248036580 de boletas para las tres elecciones - presidente de la República, diputados y senadores - y 1203192 actas electorales de casilla.

\section{Funcionarios de casillas y colaboradores}

Paralelamente al trabajo de ubicación de las casillas, se aplicó una Estrategia de Capacitación y Asistencia Electoral, aprobada por el Consejo General el 25 de julio de 2011, con la finalidad de seleccionar (a través de insaculación) y capacitar a los ciudadanos que pudieran actuar como funcionarios de casilla. Esto implica un proceso complejo conducente a asegurar que esta función se realice con entera imparcialidad y transparencia. Una vez individualizados los posibles funcionarios de casilla, se llevó adelante la primera etapa de capacitación electoral. Mediante una segunda insaculación se designó a los siete funcionarios que integrarían las mesas directivas de las casillas. Quienes no resultaron seleccionados se integraron en una lista de reserva. Entre el 9 de mayo y el 30 de junio se desarrolló la segunda etapa de capacitación electoral.

Para llevar a cabo las tareas de capacitación y asistencia electoral se requiere contratar y capacitar a numeroso personal eventual, en las categorías de capacitador asistente (CAES) y supervisor electoral.

En el proceso de 2012 intervinieron bajo estos conceptos 34327 personas: 24490 como CAES y 4837 como supervisores. 


\begin{tabular}{|c|c|c|c|c|c|c|}
\hline \multicolumn{7}{|c|}{ Personal contratado } \\
\hline \multirow{2}{*}{$\begin{array}{l}\text { Proceso } \\
\text { electoral } \\
\text { federal }\end{array}$} & \multicolumn{3}{|c|}{ SE } & \multicolumn{3}{|c|}{ CAE } \\
\hline & Mujeres & Hombres & Total & Mujeres & Hombres & Total \\
\hline \multirow{2}{*}{2006} & 1204 & 2028 & 3232 & 12244 & 11695 & 23939 \\
\hline & $37.25 \%$ & $62.75 \%$ & $100 \%$ & $51.15 \%$ & $48.85 \%$ & $100 \%$ \\
\hline \multirow{2}{*}{2009} & 1526 & 1955 & 3481 & 13299 & 12426 & 25725 \\
\hline & $43.84 \%$ & $56.16 \%$ & $100 \%$ & $51.70 \%$ & $48.30 \%$ & $100 \%$ \\
\hline \multirow{2}{*}{2012} & 2077 & 2760 & 4837 & 15635 & 13855 & 29490 \\
\hline & $42.94 \%$ & $57.06 \%$ & $100 \%$ & $53.02 \%$ & $46.98 \%$ & $100 \%$ \\
\hline
\end{tabular}

Fuente: elaboración propia con datos de los Informes Finales de la Comisión de Organización y Capacitación Electoral de los procesos electorales 2009 y 2012

A fin de asegurar el buen cumplimiento de las tareas asignadas a los CAES y a los supervisores se procedió al diseño de áreas de responsabilidad electoral (ARES), cada una de las cuales comprende un conjunto de casillas con determinada complejidad, en zona rural o urbana. Para diseñar las ARES también se calculan los tiempos y distancias entre una y otra casillas y los tiempos de traslado desde éstas a la sede del Consejo Distrital. Cinco días antes de la jornada electoral, los CAES inician la distribución de los documentos y materiales electorales a los presidentes de las mesas directivas de casilla.

Para el proceso electoral 2012, el IFE notificó y capacitó en una primera etapa a 2958060 ciudadanos, de los cuales fueron considerados aptos 2352227 (ciudadanos notificados, capacitados y que cumplían con los requisitos legales). Con esta base el IFE designó y capacitó en una segunda etapa a 1001924 funcionarios de casilla: 57.21\% mujeres, y $42.79 \%$ hombres. Se requería que en la jornada electoral estuvieran presentes los 4 propietarios, es decir, 572528 ciudadanos.

\section{Representantes de partidos políticos ante las casillas}

La legislación vigente previene que los partidos políticos tienen derecho a nombrar dos representantes propietarios y un suplente ante cada mesa directiva de casilla, así como un representante general por 
cada diez casillas electorales ubicadas en zonas urbanas y uno por cada cinco casillas rurales. Para la elección de 2012, los partidos registraron, en conjunto 1951639 ciudadanos como representantes ante mesas directivas de casillas. El siguiente cuadro muestra el total de representantes acreditados por los partidos políticos con anterioridad a la jornada electoral.

Representantes de partidos políticos acreditados ante las mesas directivas de casilla, antes de la jornada electoral

\begin{tabular}{|c|c|c|}
\hline Partido & Total representantes & $\begin{array}{c}\text { Cobertura de casillas } \\
\text { Porcentajes }\end{array}$ \\
\hline PAN & 314197 & 73.17 \\
\hline PRI & 412232 & 96.00 \\
\hline PRD & 302298 & 70.40 \\
\hline VERDE & 138118 & 32.17 \\
\hline PT & 299544 & 69.76 \\
\hline MC & 207152 & 48.24 \\
\hline NA & 278098 & 64.77 \\
\hline
\end{tabular}

Fuente: Informe Final sobre las actividades realizadas por la Comisión de Capacitación y Organización Electoral en el marco del Proceso Electoral Federal 2011-2012 (octubre de 2011 - septiembre de 2012).

\section{Observadores electorales y visitantes}

A la selección de funcionarios de casilla y al registro de representantes de partidos políticos se suma el proceso de acreditación de observadores electorales. La figura del observador electoral está regulada en el artículo 5o. del Cofipe. El 25 de agosto de 2011, el Consejo General del Instituto Federal Electoral emitió un acuerdo para fijar los lineamientos que rigen la acreditación y el desarrollo de las actividades de los observadores, conforme a la premisa de que la labor de éstos contribuye a mejorar la credibilidad, la legitimidad y la transparencia en las instituciones y los procedimientos electorales. A los observadores nacionales se agregan los visitantes extranjeros, es decir, los observadores internacionales, cuya presencia también está regulada por el Cofipe. En síntesis, para las elecciones de 2012 se acreditó a 32716 observadores y a 584 visitantes. 


\begin{tabular}{|c|c|c|c|c|}
\hline \multicolumn{5}{|c|}{ Número de observadores acreditados } \\
\hline 2000 & 2003 & 2006 & 2009 & 2012 \\
\hline 34401 & 14167 & 25321 & 18123 & 32716 \\
\hline
\end{tabular}

Fuente: Informe Final sobre las actividades realizadas por la Comisión de Capacitación y Organización Electoral en el marco del Proceso Electoral Federal 2011-2012 (octubre de 2011-septiembre de 2012).

\section{La jornada electoral}

Para dar seguimiento al desarrollo de los comicios, el IFE realizó un operativo de campo denominado Sistema de Información de la Jornada Electoral (SIJE), que suministró información permanente y oportuna tanto al Consejo General como a los consejos locales y distritales.

El sistema informó que de las 143132 casillas aprobadas, solamente dos no fueron instaladas. En el proceso de 2012 se obtuvo la cifra más alta en la instalación de casillas, en comparación con todos los procesos anteriores.

Casillas instaladas en los procesos 1991-2012

\begin{tabular}{|c|c|c|c|c|}
\hline Elección & Casillas aprobadas & $\begin{array}{c}\text { Casillas } \\
\text { instaladas }\end{array}$ & $\begin{array}{c}\text { Casillas no } \\
\text { instaladas }\end{array}$ & Porcentaje \\
\hline 1991 & 88300 & 88136 & 164 & $99.814 \%$ \\
\hline 1994 & 96415 & 96393 & 22 & $99.977 \%$ \\
\hline 1997 & 104716 & 104595 & 121 & $99.884 \%$ \\
\hline 2000 & 113423 & 113405 & 18 & $99.984 \%$ \\
\hline 2003 & 121367 & 121284 & 83 & $99.932 \%$ \\
\hline 2006 & 130488 & 130477 & 11 & $99.992 \%$ \\
\hline 2009 & 139181 & 139140 & 41 & $99.971 \%$ \\
\hline 2012 & 143132 & 143130 & 2 & 99.999 \\
\hline
\end{tabular}

Fuente: Informe Final sobre las actividades realizadas por la Comisión de Capacitación y Organización Electoral en el marco del Proceso Electoral Federal 2011-2012 (octubre de 2011-septiembre de 2012).

4 Informe final sobre las actividades realizadas por la Comisión de Capacitación y Organización Electoral en el marco del Proceso Electoral Federal 2011-2012 (octubre 2011-septiembre 2012), Instituto Federal Electoral, p. 779. 
Asimismo, el sistema permitió saber que 129101 (90.2\%) casillas estuvieron integradas por funcionarios previamente designados y capacitados, y que en 14029 (9.8\%) fue preciso incorporar como funcionarios de casilla a personas "tomadas de la fila" de ciudadanos que acudieron a sufragar. Con relación al número de funcionarios de casilla, en las 143130 casillas instaladas hubo 568948 funcionarios en total, de los cuales sólo 17971 (3.15\%) fueron "tomados de la fila" de electores que se presentaron a votar en las casillas. Asimismo, en la elección de 2012 se presentó la proporción más baja de casillas integradas por cuatro funcionarios de la fila, como se observa en el siguiente cuadro.

Cuadro comparativo de casillas instaladas con cuatro funcionarios tomados de la fila de electores

\begin{tabular}{|c|c|c|c|}
\hline Elección & Casillas instaladas & $\begin{array}{c}\text { Casillas con 4 funcionarios } \\
\text { tomados de la fila }\end{array}$ & $\%$ \\
\hline 2006 & 130477 & 52 & 0.040 \\
\hline 2009 & 139140 & 48 & 0.034 \\
\hline 2012 & 143130 & 35 & 0.024 \\
\hline
\end{tabular}

Fuente: Informe Final sobre las actividades realizadas por la Comisión de Capacitación y Organización Electoral en el marco del Proceso Electoral Federal 2011-2012 (octubre de 2011-septiembre de 2012).

Con base en la información suministrada por el SIJE y en las mismas actas de escrutinio y cómputo es posible señalar que en los comicios de 2012 estuvieron presentes 551521 representantes de partidos políticos. Para tener una visión más completa acerca de la vigilancia ejercida por los partidos el día de la elección, es preciso considerar que se contó con representantes de partidos en 141516 casillas. Esto significa el $98.87 \%$ del número total de casillas instaladas (143 130).

\section{Cobertura de casillas}

\begin{tabular}{|c|c|c|}
\hline Presencia de representantes de partidos políticos & Total de casillas & $\%$ \\
\hline Con un representante & 5937 & 4.15 \\
\hline Con 2 representantes & 17697 & 12.36 \\
\hline Con 3 representantes & 32513 & 22.72 \\
\hline
\end{tabular}




\begin{tabular}{|c|c|c|}
\hline Presencia de representantes de partidos políticos & Total de casillas & $\%$ \\
\hline Con 4 representantes & 38570 & 26.95 \\
\hline Con 5 representantes & 29253 & 20.44 \\
\hline Con 6 representantes & 13805 & 9.65 \\
\hline Con 7 representantes & 3741 & 2.61 \\
\hline Sin representantes & 1614 & 1.13 \\
\hline
\end{tabular}

Fuente: Informe final sobre las actividades realizadas por la Comisión de Capacitación y Organización Electoral en el marco del Proceso Electoral Federal 2011-2012

Por lo que se refiere a los observadores, el SIJE reveló que hubo observadores electorales en 21118 casillas.

\section{Resultados electorales}

Una vez que concluye la votación y se cierran las casillas se procede al cómputo y escrutinio de los sufragios emitidos, el traslado de los paquetes electorales a las sedes de los Consejos Distritales y la difusión de los primeros resultados. A partir del proceso electoral de 1997 se dispone de mecanismos para conocer cuanto antes los resultados electorales.

En 2012 se estableció un conteo rápido de votos, que constituye un ejercicio de estimación probabilístico, que permitió conocer las tendencias de la votación en la elección de presidente de República. Este procedimiento trabaja a partir de una muestra de casillas representativa y aleatoria, que en 2012 fueron 7567. Se actuó con el auxilio de un comité de especialistas, que asumieron la responsabilidad de generar las estimaciones estadísticas.

Los resultados del conteo rápido fueron:

Conteo rápido 2012

\begin{tabular}{|c|c|}
\hline CANDIDATOS & CONTEO RÁPIDO \\
\hline Josefina E. Vázquez Mota & $25.1 \%-26.03 \%$ \\
\hline Enrique Peña Nieto & $37.93 \%-38.55 \%$ \\
\hline Andrés Manuel López Obrador & $30.9 \%-31.86 \%$ \\
\hline Gabriel R. Quadri de la Torre & $2.27 \%-2.57 \%$ \\
\hline
\end{tabular}

Fuente: Informe final sobre las actividades realizadas por la Comisión de Capacitación y Organización Electoral en el marco del Proceso Electoral Federal 2011-2012. 
El día de los comicios entró en operación otro sistema de cómputo y difusión de resultados. Se trata del denominado Programa de Resultados Electorales Preliminares (PREP), que trabaja con sustento en los datos asentados en las actas de cómputo y escrutinio de las casillas de las tres elecciones, conforme se reciben en la sede de los Comités Distritales. En 2012, el PREP tuvo un rendimiento del 98\%: fueron capturadas 426510 actas correspondientes a las tres elecciones.

Los resultados que arrojó el PREP en lo que respecta a la elección de presidente de la República fueron:

\section{PREP 2012}

\begin{tabular}{|c|c|}
\hline CANDIDATOS & PREP \\
\hline Josefina E. Vázquez Mota & $25.40 \%$ \\
\hline Enrique Peña Nieto & $38.15 \%$ \\
\hline Andrés Manuel López Obrador & $31.64 \%$ \\
\hline Gabriel R. Quadri de la Torre & $2.30 \%$ \\
\hline
\end{tabular}

Fuente: Informe final sobre las actividades realizadas por la Comisión de Capacitación y Organización Electoral en el marco del Proceso Electoral Federal 2011-2012.

Posteriormente se llevó a cabo el cómputo distrital, realizado en los trescientos comités distritales, a los que previamente se hicieron llegar los paquetes electorales. A fin de mejorar sustancialmente la transparencia y confiabilidad de este cómputo, el Cofipe estableció nuevas causales para la apertura de paquetes y el recuento distrital de votos.

En 2009 se tuvo la primera experiencia de cómputo distrital bajo las reglas fijadas en la reforma de 2008. Fue así que se realizó el recuento de 42620 paquetes, que representaban el 30.63\% de las casillas instaladas. Entonces se trató solamente de una elección de diputados. En 2012 hubo tres elecciones y fue necesario diseñar un mecanismo que permitiera realizar el recuento de manera ininterrumpida, en el tiempo que la ley autoriza. Esto exigió un gran esfuerzo por parte de los consejos distritales, que merece amplio reconocimiento. El miércoles posterior a la jornada electoral, estos consejos llevaron adelante el recuento de 240790 paquetes electorales correspondientes a las tres elecciones, conforme a las causales establecidas en el artículo 295 del Cofipe. 
Los resultados del cómputo distrital para la elección de presidente fueron:

\section{Cómputos distritales 2012}

\begin{tabular}{|c|c|}
\hline CANDIDATOS & CÓMPUTOS DISTRITALES \\
\hline Josefina E. Vázquez Mota & $25.41 \%$ \\
\hline Enrique Peña Nieto & $38.21 \%$ \\
\hline Andrés Manuel López Obrador & $31.59 \%$ \\
\hline Gabriel R. Quadri de la Torre & $2.29 \%$ \\
\hline
\end{tabular}

Fuente: Informe final sobre las actividades realizadas por la Comisión de Capacitación y Organización Electoral en el marco del Proceso Electoral Federal 2011-2012.

Es importante señalar que se observó una coincidencia sustancial en los datos sobre la elección presidencial correspondientes al conteo rápido, al PREP y a los cómputos distritales.

$$
\begin{gathered}
\text { Resultados del PREP, el conteo rápido } \\
\text { y los cómputos distritales en la elección presidencial }
\end{gathered}
$$

\begin{tabular}{|c|c|c|c|c|}
\hline CANDIDATOS & $\begin{array}{c}\text { CONTEO } \\
\text { RÁPIDO }\end{array}$ & PREP & $\begin{array}{c}\text { CÓMPUTOS } \\
\text { DISTRITALES }\end{array}$ & $\begin{array}{c}\text { DIFERENCIA } \\
\text { PREP/ } \\
\text { CÓMPUTOS } \\
\text { PORCENTAJES }\end{array}$ \\
\hline $\begin{array}{c}\text { Josefina E. } \\
\text { Vázquez Mota }\end{array}$ & $25.1 \%-26.03 \%$ & $25.40 \%$ & $25.41 \%$ & $(-0.01)$ \\
\hline $\begin{array}{c}\text { Enrique Peña } \\
\text { Nieto }\end{array}$ & $37.93 \%-38.55 \%$ & $38.15 \%$ & $38.21 \%$ & $(-.06 \%)$ \\
\hline $\begin{array}{c}\text { Andrés Manuel } \\
\text { López Obrador }\end{array}$ & $30.9 \%-31.86 \%$ & $31.64 \%$ & $31.59 \%$ & $(+.05)$ \\
\hline $\begin{array}{c}\text { Gabriel R. Quadri } \\
\text { de la Torre }\end{array}$ & $2.27 \%-2.57 \%$ & $2.30 \%$ & $2.29 \%$ & $(+.01)$ \\
\hline
\end{tabular}

Fuente: Informe final sobre las actividades realizadas por la Comisión de Capacitación y Organización Electoral en el marco del Proceso Electoral Federal 2011-2012. 


\section{Declaración de validez y asignación de curules}

Los consejos distritales y los consejos locales, en ejercicio de sus respectivas atribuciones, entregaron a los diputados y senadores electos por el principio de mayoría relativa las correspondientes constancias. El 23 de agosto, el Consejo General declaró la validez de las elecciones de diputados y senadores por el principio de representación proporcional. La Sala Superior del Tribunal Electoral, en sesión del 28 de agosto de 2012, confirmó la asignación realizada por el Consejo General.

En total se presentaron 110 juicios de inconformidad con respecto a los resultados de la elección de diputados y 32 en lo que toca a la elección de senadores.

Los siguientes cuadros muestran la integración de ambas Cámaras:

Composición definitiva de la Cámara de Senadores

\begin{tabular}{|c|c|}
\hline & TOTAL \\
\hline PAN & 38 \\
\hline PRI & 54 \\
\hline PRD & 22 \\
\hline PT & 5 \\
\hline PVEM & 7 \\
\hline MC & 1 \\
\hline NA & 1 \\
\hline TOTAL & 128 \\
\hline
\end{tabular}

Fuente: Sistema de Información Legislativa. Segob.

Composición definitiva de la Cámara de Diputados

\begin{tabular}{|c|c|}
\hline & TOTAL \\
\hline PAN & 114 \\
\hline PRI & 213 \\
\hline PRD & 104 \\
\hline PT & 15 \\
\hline PVEM & 28 \\
\hline MC & 16 \\
\hline NA & 10 \\
\hline TOTAL & 500 \\
\hline
\end{tabular}

Fuente: Sistema de Información Legislativa, Segob. 
Un rasgo muy relevante en la integración de la Legislatura derivada de las elecciones de 2012 es el relativo a la composición de las Cámaras, considerada desde la perspectiva de género. Los siguientes números son elocuentes acerca de la aplicación de la cuota de género prevista en el Cofipe, conforme a la interpretación establecida por el Tribunal Electoral y aplicada por el IFE:

Impacto de la composición por género en la Cámara de Diputados correspondiente al periodo 1988 al 2012.

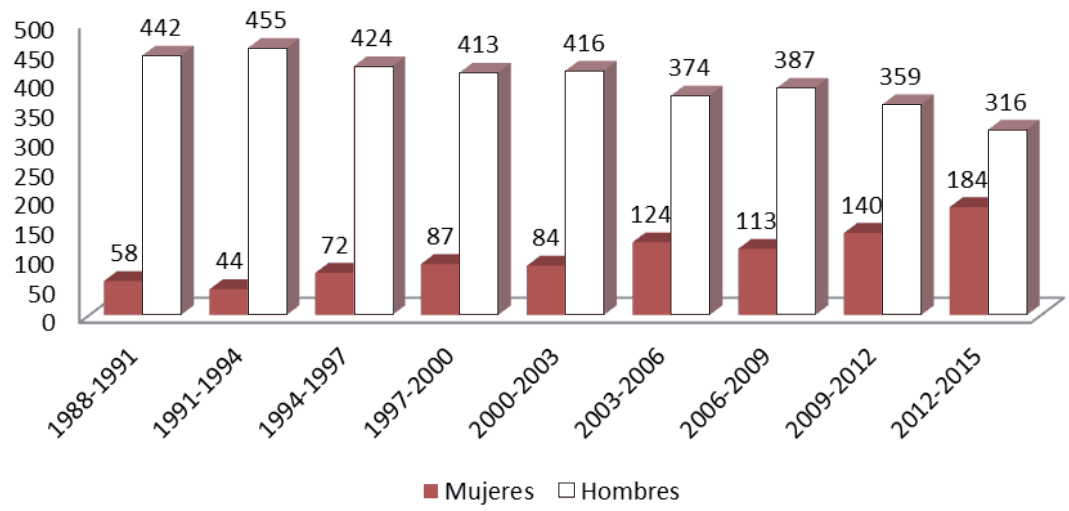

\begin{tabular}{|c|c|c|c|c|c|c|}
\hline Periodo & Legislatura & Total & Hombres & Mujeres & Mujeres & Incremento \\
\hline $\mathbf{1 9 8 8 - 1 9 9 1}$ & LIV & 500 & 442 & 58 & $11.6 \%$ & $38 \%$ \\
\hline $\mathbf{1 9 9 1 - 1 9 9 4}$ & LV & 499 & 455 & 44 & $8.8 \%$ & $-24 \%$ \\
\hline $\mathbf{1 9 9 4 - 1 9 9 7}$ & LVI & 496 & 424 & 72 & $14.5 \%$ & $64 \%$ \\
\hline $\mathbf{1 9 9 7 - 2 0 0 0}$ & LVII & 500 & 413 & 87 & $17.4 \%$ & $21 \%$ \\
\hline $\mathbf{2 0 0 0 - 2 0 0 3}$ & LVIII & 500 & 416 & 84 & $16.8 \%$ & $-3 \%$ \\
\hline $\mathbf{2 0 0 3 - 2 0 0 6}$ & LIX & 498 & 374 & 124 & $24.9 \%$ & $48 \%$ \\
\hline $\mathbf{2 0 0 6 - 2 0 0 9}$ & LX & 500 & 387 & 113 & $22.6 \%$ & $-9 \%$ \\
\hline $\mathbf{2 0 0 9 - 2 0 1 2}$ & LXI & 499 & 359 & 140 & $28.1 \%$ & $23 \%$ \\
\hline $\mathbf{2 0 1 2 - 2 0 1 5}$ & LXII & 500 & 316 & 184 & $37 \%$ & $14 \%$ \\
\hline
\end{tabular}

Fuente: Informe sobre la Evolución Normativa y las medidas afirmativas para la aplicación efectiva de las Cuotas de Género en el Proceso Electoral Federal 2011-2012, IFE, septiembre de 2012. 


\section{Declaración de validez y cómputo final de la elección de presidente}

Por lo que se refiere a la elección de presidente de la República, se presentaron 378 juicios de inconformidad, que fueron resueltos por el Tribunal Electoral. El 31 de agosto, la Sala Superior de este órgano jurisdiccional estableció el cómputo final y emitió la declaración de validez y de presidente electo de los Estados Unidos Mexicanos. Las cifras consideradas por el Tribunal fueron las siguientes:

\begin{tabular}{|c|c|c|}
\hline Candidato & Votos & Porcentaje \\
\hline Josefina Eugenia Vázquez Mota & 12732630 & $25.3 \%$ \\
\hline Enrique Peña Nieto & 19158592 & $38.2 \%$ \\
\hline Andrés Manuel López Obrador & 15848827 & $30.2 . \%$ \\
\hline Gabriel R. Quadri de la Torre & 1146085 & $2.2 \%$ \\
\hline Candidatos no registrados & 20625 & $0.04 \%$ \\
\hline Votos nulos & 1236857 & $2.4 \%$ \\
\hline Total & 50143616 & $100 \%$ \\
\hline
\end{tabular}

\section{Reflexiones finales}

En el curso del proceso electoral 2011-2012, los partidos políticos participaron en la deliberación para la toma de decisiones y ejercieron su derecho a impugnarlas, presentaron 1056 recursos contra diversos acuerdos y resoluciones, y las autoridades competentes resolvieron las controversias planteadas y dotaron de definitividad a las decisiones adoptadas. El Consejo General del IFE sesionó en 83 ocasiones: 11 sesiones ordinarias, 70 extraordinarias y una especial. ${ }^{5}$

Es preciso examinar el desarrollo del proceso electoral bajo el lente de sus cifras sobresalientes, como lo hace este artículo, que pretende informar de manera breve y concentrada. De la información reunida se desprende la dimensión de las tareas cumplidas para llevar adelante ese proceso, con participación de millares o millones de ciudadanos,

5 Información proporcionada por la Dirección del Secretariado del Instituto Federal Electoral. 
que fueron protagonistas o testigos de las actividades más relevantes y de los resultados que éstas mostraron, y en el marco de una regulación compleja que presidió el desarrollo y las incidencias del proceso electoral.

Las fuentes de esta información pueden ser consultadas detalladamente en la página web del Instituto Federal Electoral. El paso del tiempo y el análisis cuidadoso y reflexivo de los hechos y los informes correspondientes permitirán al analista $-\mathrm{y}$, en definitiva, a la sociedad en su conjunto y a los cronistas e historiadores del proceso electoral- reconocer y ponderar la dimensión del esfuerzo desplegado por los ciudadanos y por las instituciones -órganos del Estado, partidos políticos y otros actores sociales- para resolver con oportunidad y legalidad el gran reto que significaron las elecciones de 2012.

Obviamente, los números no agotan en modo alguno la indispensable reflexión acerca del desarrollo de aquel proceso. Es preciso profundizar desde diversas perspectivas y establecer, finalmente, el sentido y el alcance de este nuevo capítulo en la construcción y el fortalecimiento de la democracia. Desde luego, la democracia electoral no agota las aspiraciones de la nación mexicana. No podemos desconocer que se hallan pendientes muchos trabajos y progresos en la construcción de una democracia integral que responda a las expectativas de la nación expresadas en el artículo 3o. de la Constitución Política, como se dijo al inicio de este breve estudio. Hay preguntas que aguardan respuesta y exigencias legítimas pendientes de satisfacción.

\section{Fuentes}

\section{Legislación}

Constitución Política de los Estados Unidos Mexicanos.

Código Federal de Instituciones y Procedimientos Electorales.

Acuerdos y sentencias

CG266/2011 Acuerdo del Consejo General del Instituto Federal Electoral por el que se aprueba el Plan Integral del Proceso Electoral Federal 2011-2012. 
CG322/2012 Acuerdo del Consejo General del Instituto Federal Electoral por el que se declara que el padrón electoral y los listados nominales de electores que serán utilizados en la jornada electoral del 1 de julio del 2012, son válidos y definitivos, y que la lista nominal de lectores residentes en el extranjero es válida.

CG244/2012 Acuerdo del Consejo General del Instituto Federal Electoral por el que se emiten los lineamientos para la sesión especial de cómputo distrital del Proceso Electoral Federal 2011-2012.

Cómputo final, Calificación Jurisdiccional de la Elección, Declaración de Validez y de Presidente Electo de los Estados Unidos Mexicanos de 31 de agosto de 2012 emitido por la Sala Superior del Tribunal Electoral del Poder Judicial de la Federación.

\section{Informes}

Informe General sobre la Implementación de la Reforma Electoral durante el proceso 2008-2009. IFE, Febrero 2010.

Informe final sobre las actividades realizadas por la Comisión de Capacitación y Organización Electoral en el marco del Proceso Electoral Federal 2011-2012 (octubre 2011-septiembre 2012). IFE, 3 de octubre de 2012.

Informe sobre la Evolución Normativa y las medidas afirmativas para la aplicación efectiva de las Cuotas de Género en el Proceso Electoral Federal 2011-2012, IFE, septiembre 2012.

Informe que presenta el secretario ejecutivo del Instituto Federal Electoral con el propósito de atender el principio de definitividad que rige los procesos electorales y, por lo tanto, difundir la realización y conclusión de los actos y actividades trascendentes de este órgano electoral, en cumplimiento del Acuerdo CG331/2011, IFE, 17 de octubre de 2012. 